\title{
Indications, timing and coagulation management for tracheostomy during extracorporeal membrane oxygenation support
}

\author{
Massimo Baiocchi, Maria Benedetto, Vittorio Ferrari \\ Department of Cardiothoracic and Vascular Anaesthesia and Intensive care Sant' Orsola University Hospital, Bologna, Italy \\ Correspondence to: Massimo Baiocchi, MD. Head of Cardiothoracic and Vascular Intensive care Unit, Sant' Orsola University Hospital, Bologna, Italy. \\ Email: massimo.baiocchi@aosp.bo.it.
}

\begin{abstract}
Extracorporeal membrane oxygenation (ECMO) is used as a short-term life support in patients with cardiac or lung failure refractory to conventional treatment, as a bridge to recovery, to a long-term care system or as a bridge to transplant. Tracheostomy in these patients can be helpful, both as a respiratory weaning strategy, as well as for the cases where it is necessary to keep the mechanical support for a longer period of time. It improves lung function by reducing the dead space, it allows a faster swallowing rehabilitation and oral feeding and a more effective bronchial toilet. Furthermore, the patient can be alert and cooperative with a decrease in analgesic drug consumption and a less haemodynamic impact. Early tracheostomy appears to be associated with shorter weaning times in ECMO patients. The percutaneous technique has been proven to be superior to the surgical one in terms of reduced complications. Management of anticoagulants before performing invasive procedures in patients on ECMO is crucial. However, there are no standardised protocols. It has been shown that tracheostomised patients on ECMO require a higher amount of concentrated red blood cells. Given the link between a worse outcome and an increased blood transfusion rate it may be reasonable to conclude that bleeding in ECMO patients with tracheostomy results in a worse prognosis. However, this finding has not been confirmed in all studies.
\end{abstract}

Keywords: Tracheostomy; extracorporeal support; membrane oxygenation; extracorporeal membrane oxygenation (ECMO); veno-venous (VV) ECMO; veno-arterial (VA) ECMO

Received: 10 September 2020. Accepted: 16 December 2020.

doi: $10.21037 /$ ccts-20-154

View this article at: http://dx.doi.org/ 10.21037/ccts-20-154

\section{Introduction}

Extracorporeal membrane oxygenation (ECMO) adopts a small-scale extracorporeal technology to temporarily support the heart and /or the lung function in critically ill patients.

In the veno-venous (VV) configuration, ECMO provides a respiratory support through an extracorporeal oxygenator, while in the veno-arterial (VA) configuration it supports the cardiac function through a pump that generates a nonpulsatile flow together with an extracorporeal oxygenator.

ECMO is used as a short-term life support measure in patients with cardiac or lung failure refractory to conventional treatment, as a bridge to recovery, as a longterm care system or as a bridge to transplant. Its use in the intensive care unit has gradually increased, given the relative simplicity of pump establishment, the relatively low cost and the presence of supporting literature, especially after the H1N1 epidemic in 2009 (mainly for the VV set-up).

Tracheostomy in these patients can be helpful, both as a respiratory weaning strategy, as well as for the cases where it is necessary to keep the mechanical support for a longer period of time.

\section{Indications}

In most cases ECMO support requires the patient to be sedated, intubated and mechanically ventilated. Importantly, while in some cases it is possible to reduce sedation and 
proceed with extubation, in other cases it is possible to switch to spontaneous breathing only after having performed a tracheostomy. For example, patients awaiting for a double lung transplant can be kept awake while being on VV ECMO (awake ECMO), i.e., in spontaneous breathing (1).

This helps to avoid multi-organ deterioration linked to sedation and prolonged immobilisation while being in the transplant waiting list.

Patients on VA ECMO with good pulmonary compensation can also be kept awake whilst maintaining spontaneous ventilation, providing just light sedation in the background to tolerate the ECMO cannulae.

Patient's alertness and spontaneous breathing allow a quicker recovery of haemodynamics, a reduction in the administration of vasoactive drugs and a faster improvement in organ function (2).

Analgo-sedation in these contexts should be kept to the lowest level needed, whilst also ensuring effective pain control and prevent the onset of delirium. Benzodiazepines should be avoided due to the risk of amnesia and deterioration of space and time orientation that can easily lead to delirium.

A relatively new drug with no amnesic effects but a certain degree of analgesic effects, such as dexmedetomidine, should be preferred as prevention and management of delirium (3).

In this context, tracheostomy facilitates the respiratory weaning and the transition to spontaneous breathing. It allows to implement the awake ECMO strategy (4) even in mechanically ventilated patients, being more tolerable than the endotracheal tube.

On the contrary, severely hypoxaemic patients with ARDS on VV ECMO require deep sedation and continuous infusion of muscle relaxants in order to maintain the mechanical ventilation for a longer period. Muscle relaxation in this patients' group has proved not only to be necessary, but also useful in improving prognosis (5).

This is because tight control of lung volumes and pressures (plateau pressure, tidal volume, transpulmonary pressure), and adequate lung recruitment, alongside application of the best positive end-expiratory pressure (PEEP), helps to ensure a protective ventilation, the lowest possible mechanical power and, as a consequence, to reduce the risk of ventilator-induced lung injury (VILI).

The transition from endotracheal intubation to tracheostomy is useful in this type of patients also, both during protective mechanical ventilation and the weaning phase, facilitating the transition to spontaneous breathing and reducing the weaning time (6).
Another class of patients who requires sedation and mechanical ventilation is represented by the ones with simultaneous cardiac and pulmonary failure, undergoing peripheral VA ECMO, complicated by left ventricle overload and pulmonary oedema. It is advisable to keep them mechanically ventilated with adequate PEEP in order to avoid the onset of Harlequin syndrome, therefore ensuring adequate oxygenation of the upper part of the body not fully reached by the oxygenated blood flow from peripheral ECMO (7).

Analgosedation during ECMO, therefore, even if personalised as "light targeted" as possible, has an important impact on the already challenging haemodynamic balance, leading to a reduction in peripheral vascular resistances and in the physiological adrenergic tone.

This "iatrogenic" effect can be treated with fluid challenges (crystalloids, colloids and blood products), as well as the use of inotropic and vasoconstrictive drugs.

However, we should remember that the ECMO patient is already prone to positive fluid balances, so to ensure adequate preload and drainage into the ECMO circuit. Fluid overload, especially in the patient suffering from pulmonary failure, has been shown to increase mortality during ECMO treatment (8).

Tight fluid balance, as well as careful titration of sedatives, would therefore be appropriate.

It should also be emphasised that deep sedation only allows for passive neuromuscular physiotherapy, resulting in peripheral muscles and diaphragmatic hypotonia and critical illness coming from prolonged immobilisation.

For all these reasons, tracheostomy plays a fundamental role during ECMO.

Tracheostomy in critically ill patients has many advantages and it is widely recognised in literature.

It improves lung function by reducing the dead space. It also helps decreasing the risk of airway injury, especially with regards to vocal cords oedema due to persistent endotracheal tube.

It allows a faster swallowing rehabilitation and oral feeding and a more effective bronchial toilet.

Furthermore, the patient can be alert and cooperative, promoting better communication with healthcare providers and relatives, and a sharp decrease in sedo-analgesia drug consumption (9).

This also means less haemodynamic impact and less need of inotropic and vasoactive drugs.

Physiotherapy in awake patients promotes a faster weaning from mechanical ventilation (10), a reduction 
in the risk of ventilator-associated pneumonia (11) and a reduction in global mortality (12).

On the other hand, there are several potential risks of performing a tracheostomy: airway loss or obstruction, infection of the surgical wound, creation of a false pathway, tracheal or infraglottic stenosis, but especially in the context we are dealing with, bleeding due to the anticoagulant therapy necessary for ECMO circuit maintenance.

\section{Timing}

Tracheostomy is generally reserved to patients who require prolonged mechanical ventilation, from 24 hours up to 10 days or more. The decision to proceed should always be based on objective measures, documenting multiple unsuccessful attempts of weaning from mechanical ventilation.

There is currently no strong evidence of a reduction in mortality of critically ill patients between early $(<10$ days from endotracheal intubation) versus late $(>10$ days from endotracheal intubation) tracheostomy (12).

However, in patients requiring ECMO support, early tracheostomy appears to be associated with shorter weaning times (6).

In our centre, it is normally performed 4-7 days after endotracheal intubation when a prolonged weaning is expected or when previous extubation attempts have failed.

A surgical tracheostomy (ST) or a percutaneous technique are the two options. The latter was first introduced in 1985 by Ciaglia et al. (13), with the dilation method (percutaneous dilated tracheostomy, PDT). In recent years, PDT has gained popularity due to its simplicity and safety.

It has been proven to be superior to ST in terms of reduced complications, such as infections, major bleeding, stoma enlargement, tracheal tube displacement and death $(14,15)$.

In our centre the intensivist routinely performs a PDT using "Ciaglia" technique, under direct fibroscopic vision, to ensure the correct positioning of the tracheal access. A bleeding check and a complete bronchial toilet after the procedure are always performed via fibreoptic bronchoscopy.

\section{Bleeding and coagulation management}

Bleeding is one of the most frequent complications associated with the use of ECMO (16), although the underlying pathophysiology is still poorly investigated.
The need of anticoagulant therapy and the consumption coagulopathy that occurs due to the contact between blood and extracorporeal circuit are the major causes.

However, a certain grade of heterogeneity in the definition of bleeding still exists.

Following ELSO indications, a major bleeding would consist of a clinically overt bleeding associated with a haemoglobin $(\mathrm{Hb})$ fall of at least $2 \mathrm{~g} / \mathrm{dL}$ in 24 hours, a blood volume loss greater than $20 \mathrm{~mL} / \mathrm{kg}$ over a 24 -hour period, or a red blood cell transfusion requirement greater than $10 \mathrm{~mL} / \mathrm{kg}$ of over the same period.

A retroperitoneal, pulmonary or central nervous system bleeding, or one requiring surgical intervention would also fit the classification (17).

Several studies have already confirmed the possibility to perform percutaneous tracheostomy in patients undergoing VV or VA ECMO, with an incidence of bleeding ranging between $1.7 \%$ and $40 \%(18,19)$.

Management of anticoagulants and their possible suspension before performing invasive procedures in patients on ECMO is crucial. However, there is no standardised protocol.

An anticoagulant management tailored on the specific needs of the ECMO patients with a focus on specific procedures, is quite commonly found in literature.

Independent factors related to the risk of bleeding during ECMO according to Aubron et al. (20) are a high aPTT, an APACHE score $\geq$ III and ECMO institution subsequent to surgical complication.

Point of care tests do not appear to be significant in predicting the risk of bleeding before an invasive procedure.

However, patients with major bleeding have shown an elongated InTEM CT and an elongated FibTEM Clot formation time (CFT), i.e., a delay in the activation of the intrinsic coagulation pathway (21).

Two studies found the same number of major bleeds when performing a tracheostomy while on ECMO with a short withdrawal of anticoagulant versus a longer suspension.

However, in both patient groups they did not reach the anticoagulation target, with values of aPTT/INR of 1.4 and 1.3 , respectively $(18,22)$.

A low post-oxygenating $\mathrm{PaO}_{2}$ has also been seen in patients who develop major complications including bleeding after percutaneous tracheostomy. One possible hypothesis is that there could be a dysfunction in the circuit causing a circuit related disseminated intravascular coagulopathy (DIC) that is unmasked during the 
tracheostomy (22).

A longer use of the ECMO circuit and a higher transfusion rate could explain this dysfunction.

The platelet activity and the platelet count are fundamental for adequate haemostasis during ECMO, especially when undertaking invasive procedures.

A platelet count greater than $100 \times 10^{9} / \mathrm{L}$, as recommended by the guidelines, is present in all analysed studies, including those patients who develop major bleedings. There are no significant differences in the platelet count between the patients who develop major bleeding and those who do not $(18,19)$.

Bleeding in a patient on ECMO, not just related to tracheostomy, is associated with increased mortality (20).

It has also been shown that tracheostomised patients on ECMO require a higher amount of concentrated red blood cells.

Given the link between a worse outcome and an increased blood transfusion rate in different patients' groups, including those on ECMO (23), it may be reasonable to conclude that bleeding in ECMO patients with tracheostomy results in a worse prognosis, even if this finding is not confirmed in other studies (22).

Nevertheless, it has been shown that patients who have developed complications from tracheostomy require more days of ECMO care (22).

\section{Discussion}

The usefulness of tracheostomy during ECMO therapy has not been confirmed in literature. Furthermore, the evidence of a higher amount of blood products needed could bring us to conclude that tracheostomy during ECMO is linked to a worse prognosis.

However, in our routine clinical practice there is a strong evidence of benefits coming from early percutaneous tracheostomy in patients on $\mathrm{VV}$ ECMO, in terms of respiratory weaning strategy as well as in terms of pulmonary infection rate.

There could be some concerns regarding early tracheostomy in patients on peripheral or central VA ECMO, due to the generally higher amount of anticoagulation needed and the generally shorter period of treatment. To conclude, there is no gold standard regarding indications and timing of tracheostomy during ECMO. Clinical decision should be tailored on the specific patients' needs.

\section{Acknowledgments}

Funding: None.

\section{Footnote}

Provenance and Peer Review: This article was commissioned by the Guest Editors (Andrea Dell'Amore and Nizar Asadi) for the series "Mechanical Extracorporeal CardioRespiratory Supports in General Thoracic Surgery" published in Current Challenges in Thoracic Surgery. The article was sent for external peer review organized by the Guest Editors and the editorial office.

Conflicts of Interest: The authors have completed the ICMJE uniform disclosure form (available at http:// dx.doi.org/10.21037/ccts-20-154). The series "Mechanical Extracorporeal Cardio-Respiratory Supports in General Thoracic Surgery" was commissioned by the editorial office without any funding or sponsorship. The authors have no other conflicts of interest to declare.

Ethical Statement: The authors are accountable for all aspects of the work in ensuring that questions related to the accuracy or integrity of any part of the work are appropriately investigated and resolved.

Open Access Statement: This is an Open Access article distributed in accordance with the Creative Commons Attribution-NonCommercial-NoDerivs 4.0 International License (CC BY-NC-ND 4.0), which permits the noncommercial replication and distribution of the article with the strict proviso that no changes or edits are made and the original work is properly cited (including links to both the formal publication through the relevant DOI and the license). See: https://creativecommons.org/licenses/by-nc-nd/4.0/.

\section{References}

1. Mohite PN, Sabashnikov A, Reed A, et al. Extracorporeal life support in "awake" patients as a bridge to lung transplant. Thorac Cardiovasc Surg 2015;63:699-705.

2. Mojoli F, Venti A, Pellegrini C, et al. Hospital survival and long term quality of life after emergency institution of venoarterial ECMO for refractory circulatory collapse. Minerva Anestesiol 2013;79:1147-55. 
3. Pasero D, Sangalli F, Baiocchi M, et al. Experienced use of dexmedetomidine in the Intensive Care Unit: A report of a structured consensus. Turk J Anaesthesiol Reanim 2018;46:176-83.

4. Swol J, Strauch JT, Schildhauer TA. Tracheostomy as a bridge to spontaneous breathing and awake-ECMO in non-transplant surgical patients. Eur J Heart Fail 2017;19:120-3.

5. Hraiech S, Yoshida T, Papazian L. Balancing neuromuscular blockade versus preserved muscle activity. Curr Opin Crit Care 2015;21:26-33.

6. MacLaren G, Combes A, Bartlett RH. Contemporary extracorporeal membrane oxygenation for adult respiratory failure: life support in the new era. Intensive Care Med 2012;38:210-20.

7. Shekar K, Roberts JA, Ghassabian S, et al. Sedation during extracorporeal membrane oxygenation-why more is less. Anaesth Intensive Care 2012;40:1067-9.

8. Schmidt M, Bailey M, Kelly J, et al. Impact of fluid balance on outcome of adult patients treated with extracorporeal membrane oxygenation. Intensive Care Med 2014;40:1256-66.

9. Nieszkowska A, Combes A, Luyt CE, et al. Impact of tracheotomy on sedative administration, sedation level, and comfort of mechanically ventilated intensive care unit patients. Crit Care Med 2005;33:2527-33.

10. Arabi Y, Haddad S, Shirawi N, et al. Early tracheostomy in intensive care trauma patients improves resource utilization: a cohort study and literature review. Crit Care 2004;8:R347-52.

11. Heffner JE, Hess D. Tracheostomy management in the chronically ventilated patient. Clin Chest Med 2001;22:55-69.

12. Andriolo BN, Andriolo RB, Saconato H, et al. Early versus late tracheostomy for critically ill patients. Cochrane Database Syst Rev 2015;1:CD007271.

13. Ciaglia P, Firsching R, Syniec C. Elective percutaneous dilatational tracheostomy: a new simple bedside procedure: preliminary report. Chest 1985;87:715-9.

doi: $10.21037 /$ ccts-20-154

Cite this article as: Baiocchi $M$, Benedetto $M$, Ferrari $\mathrm{V}$. Indications, timing and coagulation management for tracheostomy during extracorporeal membrane oxygenation support. Curr Chall Thorac Surg 2021.
14. Johnson-Obaseki S, Veljkovic A, Javidnia H. Complication rates of open surgical versus percutaneous tracheostomy in critical ill patients. Laryngoscope 2016;126:2459-67.

15. Delaney A, Bagshaw SM, Nalos M. Percutaneous dilatational tracheostomy versus surgical tracheostomy in critically ill patients: a systematic review and meta-analysis. Crit Care 2006;10:R55.

16. Zangrillo A, Landoni G, Biondi-Zoccai G, et al. A metaanalysis of complications and mortality of extracorporeal membrane oxygenation. Crit Care Resusc 2013;15:172-8.

17. Extracorporeal Life Support Organization. ELSO Anticoagulation guideline. Ann Arbor, MI USA 2014. p. 17. Available online: https://www.elso.org/ Portals/0/Files/ elsoanticoagulationguideline8-2014-table-contents.pdf

18. Kruit N, Valchanov K, Blaudszun G, et al. Bleeding Complications Associated With Percutaneous Tracheostomy Insertion in Patients Supported With Venovenous Extracorporeal Membrane Oxygen Support: A 10-Year Institutional Experience. J Cardiothorac Vasc Anesth 2018;32:1162-6.

19. Braune S, Kienast S, Hadem J, et al. Safety of percutaneous dilatational tracheostomy in patients on extracorporeal lung support. Intensive Care Med 2013;39:1792-9.

20. Aubron C, DePuydt J, Belon F, et al. Predictive factors of bleeding events in adults undergoing extracorporeal membrane oxygenation. Ann Intensive Care 2016;6:97.

21. Hellmann C, Schmutz A, Kalbhenn J. Bleeding during veno-venous ECMO cannot reliably be predicted by rotational thrombelastometry (ROTEM ${ }^{\mathrm{TM}}$ ). Perfusion. 2018;33:289-96.

22. Dimopoulos S, Joyce H, Camporota L, et al. Safety of Percutaneous Dilatational Tracheostomy During VenoVenous Extracorporeal Membrane Oxygenation Support in Adults With Severe Respiratory Failure. Crit Care Med 2019;47:e81-8.

23. Mazzeffi M, Greenwood J, Tanaka K, et al. Bleeding, transfusion, and mortality on extracorporeal life support: ECLS Working Group on Thrombosis and Hemostasis. Ann Thorac Surg 2016;101:682-9. 